\title{
Persistence of immunity after vaccination with a capsular group B meningococcal vaccine in 3 different toddler schedules
}

\author{
Manish Sadarangani BM BCh DPhil, Tim Sell MBChB, Mildred A. Iro MBBS PgDip, Matthew D. Snape MBBS MD, \\ Merryn Voysey MBiostat, Adam Finn MA BM BCh PhD, Paul T. Heath MBBS, Gianni Bona MD, \\ Susanna Esposito MD, Javier Diez-Domingo MD PhD, Roman Prymula MD PhD, Adefowope Odueyungbo PhD, \\ Daniela Toneatto MD, Andrew J. Pollard MBBS PhD; for the European MenB Vaccine Study Group*
}

Cite as: CMAJ 2017 October 16;189:E1276-85. doi: 10.1503/cmaj.161288

\section{ABSTRACT \\ BACKGROUND: One schedule for the cap- sular group $B$ meningococcal vaccine $4 C M e n B$ is 2 doses that are administered 2 months apart for children aged 12-23 months, with a booster dose 12-24 months later. Our objective was to provide data on persistence of human serum bactericidal antibody (hSBA) titres in children up to 4 years of age after initial doses at 12-24 months, and immunoge- nicity of a booster dose at 48 months of age compared with vaccine-naive children.}

METHODS: Children previously immunized, as part of a randomized controlled trial, with 2 doses of $4 C M e n B$ vaccine at 12-24 months of age received a booster at
4 years of age. Vaccine-naive age-matched toddlers received 2 doses of 4 CMenB. Human serum bactericidal antibody titres against reference strains H44/76, 5/99, NZ98/254 and M10713 were evaluated before and after innoculation with $4 \mathrm{CMenB}$ vaccine in 4-year-old children.

RESULTS: Of 332 children in the study, 123 had previously received $4 \mathrm{CMen} B$ and 209 were vaccine-naive controls. Before the booster, the proportions of participants (previously vaccinated groups compared with controls) with hSBA titres of $1: 5$ or more were as follows: $9 \%-11 \%$ v. $1 \%$ (H44/76), $84 \%-100 \%$ v. $4 \%$ (5/99), $0 \%-18 \%$ v. $0 \%(N Z 98 / 254)$ and $59 \%-60 \%$ v. $60 \%$ (M10713). After 1 dose of 4 CMenB in previously immunized children, the proportions of participants achieving hSBA titres of $1: 5$ or more were $100 \%$ (H44/76 and 5/99), 70\%-100\% (NZ98/254) and $90 \%-100 \%$ (M10713).

INTERPRETATION: We found that waning of hSBA titres by 4 years of age occurred after 2 doses of $4 C M e n B$ vaccine administered at 12-24 months, and doses at 12-24 months have a priming effect on the immune system. A booster may be necessary to maintain hSBA titres of $1: 5$ or more among those children with increased disease risk. Trial registration: ClinicalTrials.gov, no. NCT01717638
$\mathbf{N}$ eisseria meningitidis causes meningitis and septicemia, ${ }^{1}$ with rapid disease onset, high rate of case fatality ${ }^{2,3}$ and increased rates of long-term neurologic and nonneurologic sequelae. ${ }^{4-11}$ Over the last 15 years, conjugate vaccines have resulted in near elimination of endemic disease in countries with high coverage group $C$ vaccine programs ${ }^{12}$ and marked effect of group A vaccines in Africa. ${ }^{13}$ In many countries, most disease is now caused by capsular group B N. meningitidis (MenB).$^{14}$ It is the leading cause of meningococcal disease in Canada, with a peak incidence of 6.16 cases per 100000 per year in children less than 1 year of age..$^{15} \mathrm{~A}$ recently licensed vaccine (4CMenB) designed primarily to prevent MenB infection was introduced into the routine infant immunization schedule in the United Kingdom in September $2015,{ }^{16}$ and was used in a response to hyperendemic MenB dis- ease in Quebec, Canada. ${ }^{17}$ Although routine use of the vaccine is intended for immunization of infants, the vaccine may be considered in older children for catch-up campaigns or outbreaks. Therefore, information on duration of protection is needed.

One recommended schedule for 4 CMenB is 2 doses that are administered at least 2 months apart for children aged 12-23 months, followed by a booster dose 12-23 months later, because a single dose does not result in sufficient production and persistence of protective antibodies. ${ }^{18}$ Different dosing schedules are used at different ages. Infants require additional doses, ${ }^{18}$ and shorter intervals have been used in outbreak settings. ${ }^{19}$ We know of no data on the persistence of bactericidal antibodies (on which the correlate of protection is based) through to the pre-school period following vaccination of toddlers. 
We aimed to assess the persistence of human serum bactericidal antibody (hSBA) titres in children who were 4 years of age and previously vaccinated with 2 doses of 4 CMenB in the second year of life compared with age-matched children who were vaccine naive, the percentage of children with hSBA titres of $1: 5$ or more after a booster dose of $4 \mathrm{CMenB}$ at 4 years of age, the proportion of vaccine-naive 4-year-old children achieving hSBA titres of $1: 5$ or more after 2 doses of $4 C M e n B$ and adverse reactions in 4-year-old children after vaccination with 4CMenB.

\section{Methods}

\section{Study type and setting}

We conducted an open-label multicentre extension (NCT01717638) to a randomized controlled trial (RCT) conducted at 31 centres in the Czech Republic, Italy, Spain and the UK between November 2012 and October 2013 (Appendix 1, supplementary Table 1, available at www.cmaj.ca/lookup/suppl/ doi:10.1503/cmaj.161288/-/DC1).

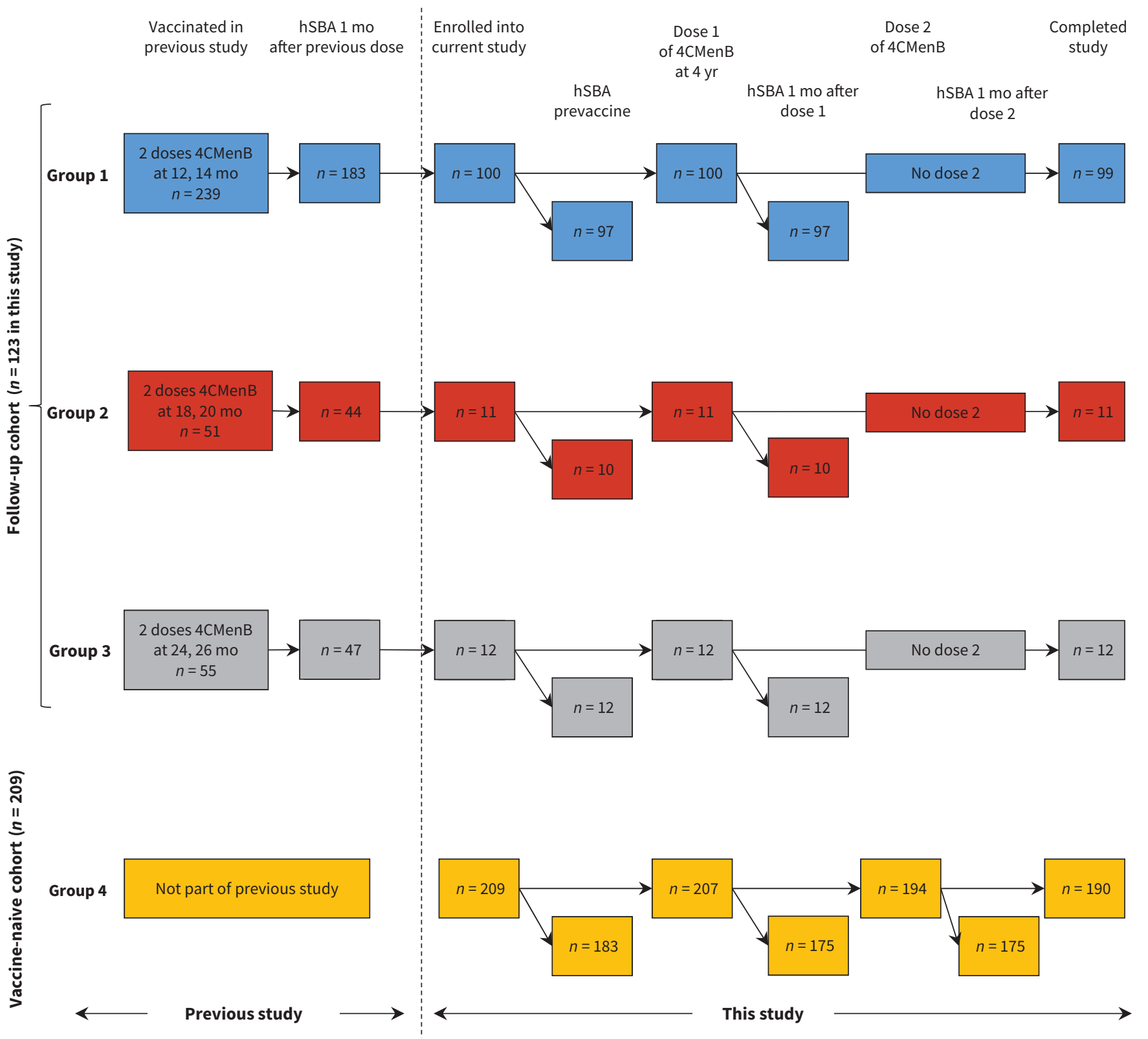

Figure 1: Flow diagram for selection of participants. All participants in the follow-up cohort received 2 previous doses of 4 CMenB vaccine at 12 and 14 mo (Group 1), 18 and 20 mo (Group 2) or 24 and 26 mo (Group 3) in a previous study. ${ }^{21}$ The vaccine-naive cohort had not received any $4 C M e n B$ doses previously. All children received 1 dose at $4 \mathrm{yr}$ of age; the vaccine-naive cohort were given an additional dose 2 mo later. Blood samples for persistence analysis were taken before any doses were given at $4 \mathrm{yr}$ of age (prevaccine time point) and for booster analysis $30 \mathrm{~d}$ after each dose. Safety data were collected after each dose. Time points where blood samples were taken for measurement of hSBA are shown slightly offset from the pain participant flow because inability to measure SBA did not preclude ongoing involvement of the participant in the study (i.e., this did not result in withdrawal or loss to follow-up). hSBA = human serum bactericidal antibody, $4 \mathrm{CMenB}=$ multicomponent meningococcal serogroup $B$ vaccine. 


\section{Study participants}

This study was part of a larger study of which the primary outcome was persistence of hSBA titres in infants given 3 priming doses of 4 CMenB vaccine as infants and a booster dose at 12, 18 or 24 months, and reported elsewhere..$^{20}$

We invited participants who were involved in a previous followup study to take part in this study via an invitation letter. In the previous study, 3 cohorts received 2 doses of 4 CMenB at 12 and 14 months $(n=239), 18$ and 20 months $(n=51)$ or 24 and 26 months $(n=55) .{ }^{21}$ Of these, 123 participants were enrolled as the follow-up cohort for our study. We also recruited 209 participants who were vaccine naive as age-matched controls (Figure 1). We included participants if they met the following criteria: child 48-59 months of age and healthy, and who had received 2 doses of $4 \mathrm{CMenB}$ in the previous study ("follow-up" participants) or had not received doses of 4 CMenB ("vaccine-naive" participants). We excluded participants if they had previously ascertained or suspected disease caused by $N$. meningitidis, had household contact or intimate exposure to an individual with laboratory-confirmed $N$. meningitidis, had a previous allergic reaction to any vaccine component, had a serious chronic or progressive disease, had known or suspected immunosuppression, had participated in another clinical trial within 90 days of pre-enrolment or during the study, or was a family member of the research staff.

\section{Vaccine}

The $4 \mathrm{CMenB}$ vaccine used in this study (Bexsero, GSK) contained $50 \mu \mathrm{g}$ each of 3 proteins (Neisseria heparin binding antigen [NHBA], Neisserial adhesin $\mathrm{A}[\mathrm{NadA}]$ and factor $\mathrm{H}$-binding protein [ $\mathrm{fHbp}]$ ], $25 \mu \mathrm{g}$ of outer membrane vesicle from $\mathrm{N}$. meningitidis strain NZ98/254 and aluminum hydroxide..$^{18}$ All participants were innoculated with $0.5 \mathrm{~mL}$ of vaccine intramuscularly.

\section{Interventions}

Follow-up participants received 1 dose of $4 \mathrm{CMenB}$ vaccine, and we obtained blood samples before and 30 days after vaccination. Participants who were vaccine naive received 2 doses of 4 CMenB that we administered 2 months apart; we obtained blood samples before the first innoculation and 1 month after each dose.

We asked parents to record adverse events for 7 days after vaccination; we then graded the severity of the adverse events that were recorded. Solicited local adverse events were injection site pain, erythema, induration and swelling. Solicited systemic adverse events were fever (axillary temperature $\geq 38^{\circ} \mathrm{C}$ ), change in eating habits, sleepiness, vomiting, diarrhea, irritability, arthralgia, headache and rash. Adverse events requiring a physician's visit and use of antipyretics or analgesia were recorded. We determined the relation of adverse events to the study vaccine by considering temporal relations and biological plausibility.

\section{Assessment of serum bactericidal antibody}

We assessed immunogenicity by measuring hSBA titres, using human serum as the source of exogenous complement. ${ }^{22}$ Assays were performed at Novartis Vaccines and Diagnostics $(\mathrm{GmbH}$, Marburg, Germany). The fHbp response was assessed with strain H44/76, NadA with strain 5/99, PorA with NZ98/254 and NHBA with M10713.

\section{Statistical analysis}

We calculated the percentage of participants with hSBA titres of 1:5 or more at each time point of blood sampling, and associated 2-sided 95\% exact Clopper-Pearson confidence intervals (Cls) were calculated for each indicator strain. We assumed that an interpolated hSBA titre of 1:5 or more represented $95 \%$ confi-

Table 1: Participant characteristics

\section{Characteristic}

Ages at previously administered 4 CMenB doses, mo

No. of doses administered

Age at administration of first dose, mo; mean \pm SD

Male sex (\%)

White (\%)

Weight, kg; mean \pm SD

Height, $\mathrm{cm}$; mean \pm SD

\section{No. of participants in follow-up cohort (\%)*}

$\begin{array}{ccc}\text { Group } 1 & \text { Group 2 } & \text { Group } 3 \\ n=100 & n=11 & n=12\end{array}$

12,14

1

$51.7 \pm 3.3$

18,20

1

$50(50.0)$

$97(97.0)$

$18.1 \pm 2.4 \dagger$

$106 \pm 4$
$53.4 \pm 4.3$

5 (45)

8 (73)

$18.7 \pm 2.4 \ddagger$

$107 \pm 4$
24,26

$56.8 \pm 1.5$

$8(67)$

$7(58)$

$18.8 \pm 1.8 \S$

$108 \pm 6$
No. of participants

in vaccine-naive cohort (\%)*

\section{Group 4}

$n=209$

NA

2 (2 mo apart)

$53.7 \pm 3.6$

$110(53)$

193 (92)

$18.1 \pm 2.5$

$107 \pm 5^{\star \star}$

Note: $4 \mathrm{CMenB}=$ capsular group $\mathrm{B}$ meningococcal vaccine, $\mathrm{hSBA}=$ human serum bactericidal antibody, $\mathrm{NA}=$ not applicable, $\mathrm{SD}=$ standard deviation. $\mathrm{All}$ participants in the follow-up cohort received 2 previous doses of 4CMenB vaccine at 12 and 14 mo (Group 1), 18 and 20 mo (Group 2) or 24 and 26 mo

(Group 3) in a previous study. ${ }^{21}$ Group 4 included participants with no previous 4CMenB vaccination.

*Unless specified otherwise.

†Denominator used: $n=91$

$\ddagger$ Denominator used: $n=9$.

§Denominator used: $n=11$

IDenominator used: $n=189$

${ }^{\star \star}$ Denominator used: $n=205$ 
dence that participants achieving this titre had an hSBA titre of 1:4 or more. A postvaccine hSBA titre of 1:4 or more is currently accepted as the presumed protective threshold against meningococcal disease. ${ }^{23}$ We calculated hSBA geometric mean titres (GMTs). Geometric mean ratios (GMRs) were determined by comparison of postvaccination and prevaccination values of GMT. We computed values of GMT and GMR with associated $95 \%$ Cls by taking anti-logs. Sample size for the follow-up participants was determined by the number of participants in the previous study whose parents were willing to take part. Although our primary aim was descriptive, the secondary aim in the vaccine-naive cohort was to show a "sufficient" immune response following 2 doses of the vaccine, which we predefined as more than $70 \%$ of participants with hSBA titres of 1:5 or more. Power calculations assumed that the actual percentage would be $80 \%$; therefore, 162 participants were required in the vaccine-naive cohort for $79 \%$ power $(5 \% \alpha)$ to show a sufficient immune response against NZ98/254, and providing more than $99.9 \%$ power for strains $\mathrm{H} 44 / 76$ and $5 / 99$. Assuming a $15 \%$ dropout rate, we calculated that an enrolment of 190 participants in the vaccine-naive control group was required.

\section{Ethics approval}

Written, informed consent was obtained from parents or legal guardians of the participants. Ethical approval was obtained from independent review committees at all study centres.
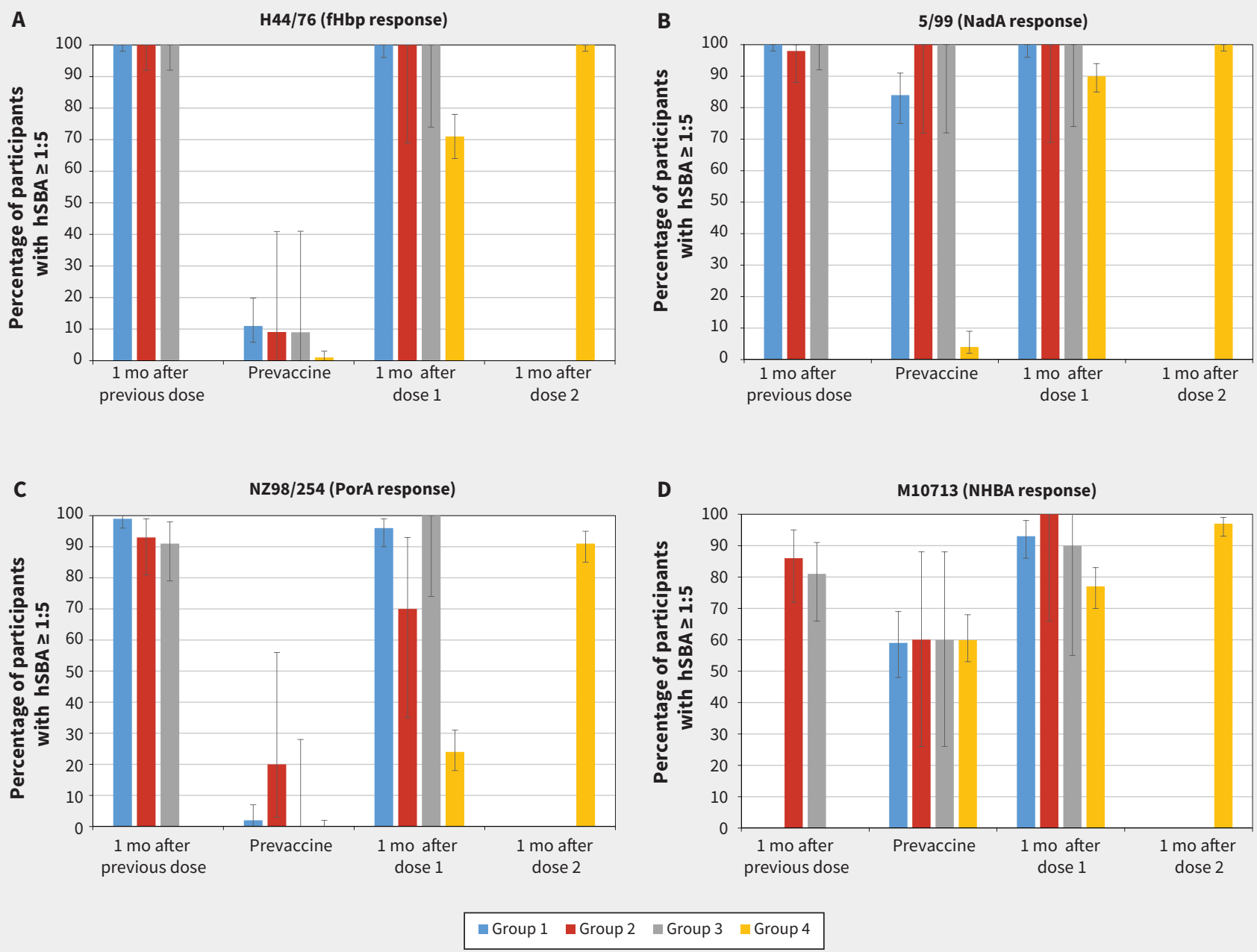

Figure 2: Proportion of participants with hSBA titres $\geq 1: 5$ at each time point against each strain. Proportion of participants with hSBA titres $\geq 1: 5$ at 1 mo after the previous dose (Groups 1, 2 and 3 only) is based on data from Snape and colleagues. ${ }^{21}$ Time points from this study include prevaccine (before first dose, all groups), 1 mo postdose 1 (all groups) and 1 mo postdose 2 (Group 4 only). The prevaccine category for Groups 1,2 and 3 represents persistence of hSBA following the vaccine doses at 12, 18 or 24 mo. Participants in Group 1 were not tested for M10713 strains at 1 mo following the previous dose in the prior study because the strain was not available. Bars represent overall percentage of participants achieving an hSBA titre $\geq 1: 5$, with error bars representing 95\% confidence intervals. Data are shown separately for the 4 indicator strains: (A) $\mathrm{H} 44 / 76$ (fHbp response), (B) 5/99 (NadA response), (C) NZ98/254 (PorA response) and (D) M10713 (NHBA response). All participants in the follow-up cohort received 2 previous doses of 4 CMenB vaccine at 12 and 14 mo (Group 1), 18 and 20 mo (Group 2) or 24 and 26 mo (Group 3) in a previous study. ${ }^{21}$ Group 4 included participants with no previous $4 \mathrm{CMenB}$ vaccination. $\mathrm{fHbp}=$ factor $\mathrm{H}$-binding protein, $\mathrm{hSBA}=$ human serum bactericidal antibody, NadA = Neisserial adhesin $\mathrm{A}$, $\mathrm{NHBA}=$ Neisseria heparin binding antigen, Por $\mathrm{A}=$ porin $\mathrm{A}$. 


\section{Results}

\section{Study population}

Of the 304 children who were invited to participate, 123 were enrolled into the follow-up cohort: 100 received their first dose of 4CMenB vaccine at 12 months (Group 1), 11 at 18 months (Group 2) and 12 at 24 months (Group 3); 122 of 123 participants (99\%) completed the study (Figure 1). The vaccine-naive cohort (Group 4) included 209 children, of whom 190 (91\%) completed the study. The demographic characteristics of the groups were similar (Table 1).

\section{Immunogenicity}

Persistence of hSBA titres of 1:5 or more in participants at 4 years of age in the follow-up cohort (represented by the "prevaccine" category) was $9 \%-11 \%$ against $\mathrm{H} 44 / 76,84 \%-100 \%$ against $5 / 99$, $0 \%-18 \%$ against NZ98/254 and 59\%-60\% against M10713 (Figure 2). In all groups, hSBA GMTs were less than 5 for H44/76 and NZ98/254 but were 5 or more for 5/99 and M10713 (Figure 3). After the third dose of 4 CMenB vaccine at 4 years of age, $70 \%$ $100 \%$ of participants had an hSBA titre of 1:5 or more, depending on the target strain (Figure 2). Values of hSBA GMTs after the
A

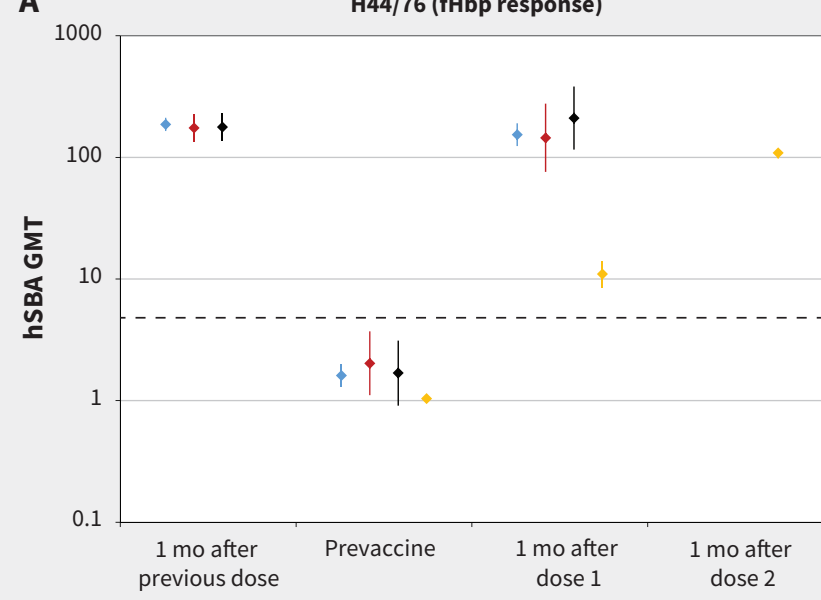

C

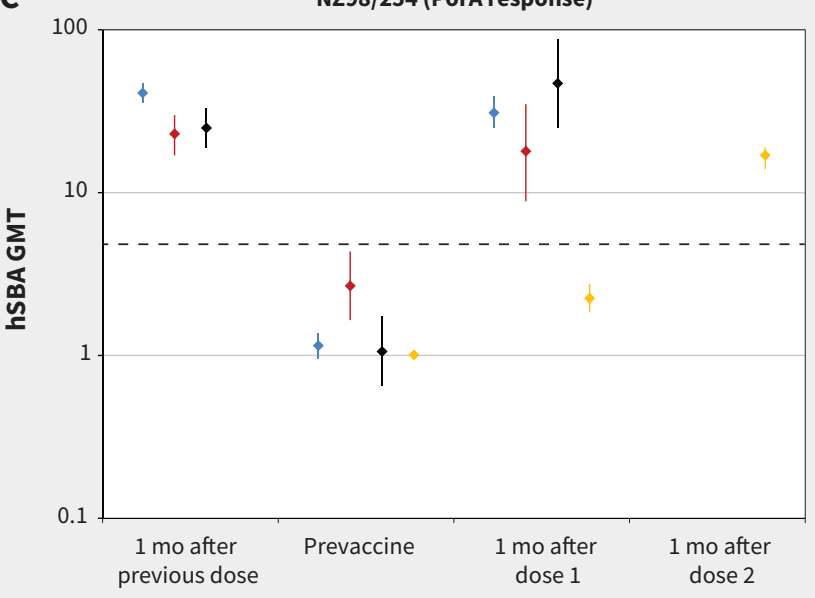

B

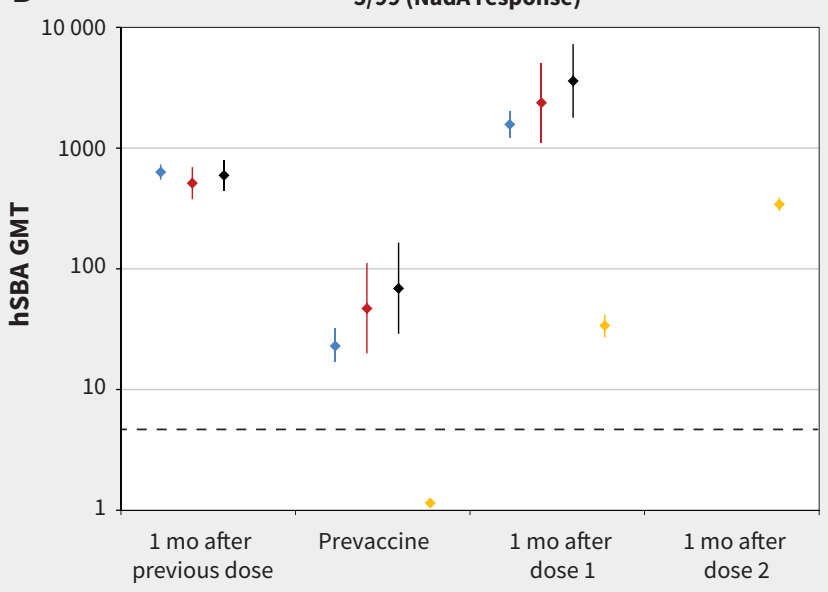

D

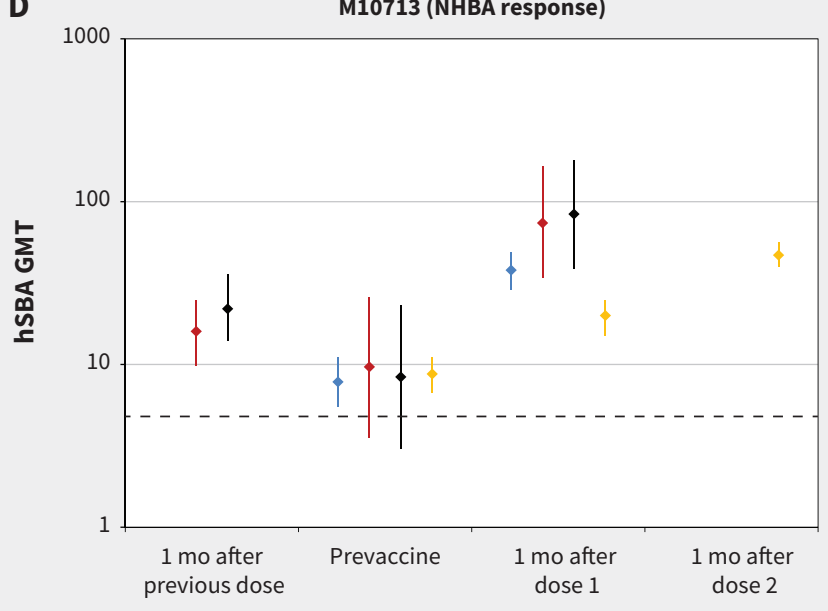

Figure 3: Geometric mean titres for hSBA at each time point against each strain. Values of geometric mean titres at 1 mo after the previous dose (Groups 1, 2 and 3 only) are based on data from Snape and colleagues. ${ }^{21}$ Time points from this study include prevaccine (before first dose, all groups), 1 mo postdose 1 (all groups) and 1 mo postdose 2 (Group 4 only). Participants in Group 1 were not tested for M10713 strains at 1 mo after the previous dose in the prior study because the strain was not available. Points represent overall GMT, with error bars representing $95 \%$ confidence intervals. Data are shown separately for the 4 indicator strains: (A) H44/76 (fHbp response), (B) 5/99 (NadA response), (C) NZ98/254 (PorA response) and (D) M10713 (NHBA response). Human serum bactericidal antibody titre of 1:5 shown as horizontal dashed line on each graph to enable comparison as different $\mathrm{Y}$ axis scale on each graph. All participants in the follow-up cohort received 2 previous doses of 4 CMenB vaccine at 12 and 14 mo (Group 1 ), 18 and 20 mo (Group 2) or 24 and 26 mo (Group 3) in a previous study. ${ }^{21}$ Group 4 included participants with no previous $4 \mathrm{CMenB}$ vaccination. $\mathrm{fHbp}=$ factor $\mathrm{H}-$ binding protein, GMT = geometric mean titre, hSBA = human serum bactericidal antibody, NadA = Neisserial adhesin A, NHBA = Neisseria heparin binding antigen, PorA = porin $\mathrm{A}$. 
third dose were similar to or higher than the GMTs that were achieved 1 month after the second dose (Figure 3). The GMRs that compared responses before and after administration of booster vaccine were highest for strain H44/76 (67-133) and lowest for strain M10713 (5.24-7.35) (Table 2).

In the vaccine-naive cohort, $0 \%-5 \%$ of participants had hSBA titres of $1: 5$ or more against H44/76, 5/99 and NZ98/254 before vaccination, and $60 \%$ against M10713 (Figure 2). After 2 doses in these previously unvaccinated participants, 91\%$100 \%$ achieved an hSBA titre of $1: 5$ or more, with a 4 -fold rise from baseline in $51 \%-100 \%$ of participants, depending on the target strain (Appendix 1, supplementary Figure 1). Values of GMTs after 2 doses were lower or similar to values after the booster dose in the follow-up groups (Figure 3). Values of GMRs were similar or higher after 2 doses compared with after the booster dose in the follow-up groups; the highest values were observed with strain 5/99 $($ GMR $=299)$ and lowest with M10713 (GMR = 5.12).

\section{Reactogenicity}

The most commonly reported local adverse event was pain at injection site, which occurred in 114 of 121 (94\%) participants in the follow-up cohort overall after the booster dose, and in 185 of 205 (90\%) participants in the vaccine-naive cohort after the first dose and in 157 of 194 (81\%) after the second dose (Table 3). Sleepiness and irritability were the most common systemic adverse events overall, occurring after 200 of 519 (39\%) and 188 of $518(36 \%)$ doses, respectively (Table 3$)$. Fever $\left(\geq 38.0^{\circ} \mathrm{C}\right)$ occurred in 25 of 121 (21\%) participants in the follow-up cohort after a single dose, and in 20 of 204 (10\%) participants in the vaccine-naive cohort after the first dose and 16 of 189 (8\%) after the second dose (Table 3). Treatment for fever was given after 73 of $518(14 \%)$ vaccine doses overall, preventive therapy before 49 of $517(9 \%)$ doses, and medical attention for fever was sought in 9 of $517(2 \%)$ cases (Table 3).
There were 3 reported serious adverse events that resulted in admission to hospital, all of which occurred in participants in the vaccine-naive cohort. None were considered vaccine-related. Croup developed in 1 child 60 days after the first dose; another child had a head injury with concussion, contusion and periorbital hematoma 23 days after the first dose; a third child required intravenous fluids for gastroenteritis and dehydration 3 days after the second dose of vaccine. There was 1 withdrawal (parental decision) because of an adverse event (moderate injection site pain after the first vaccination in a child in the vaccinenaive cohort).

\section{Interpretation}

Our study showed waning of bactericidal antibodies in children 2 years or more of age after vaccination with 4 CMen $B$ in the second year of life, which suggests that additional booster doses may be needed if protection is required beyond 4 years of age. A single booster dose at 4 years of age was sufficient to boost hSBA titres to protective levels in most children who were previously vaccinated, and 2 doses in vaccine-naive children provided similar protection. This supports the current licensed schedule in this age group. Although hSBA titre is accepted as the correlate of protection against meningococcal disease and used for vaccine licensure, vaccine effectiveness at a population level can be assessed only after vaccination. In the UK, introduction of $4 \mathrm{CMenB}$ vaccine into the infant schedule resulted in $83 \%$ effectiveness, with prior in vitro immunogenicity data predicting coverage of $73 \%-88 \% .^{24}$

One previous study in Europe found variable waning of bactericidal antibodies in the first 12 months after 2 doses of 4 CMenB given at 12 and 14 months or 13 and 15 months. ${ }^{25}$ After 12 months, $56 \%-75 \%$ of participants had hSBA titres of $1: 5$ or more against $\mathrm{H} 44 / 76,94 \%-97 \%$ against $5 / 99$ and $6 \%-18 \%$ against NZ98/254.

Table 2: Geometric mean ratios of human serum bactericidal antibody titres in participants before vaccination and after vaccination for different indicator strains, by cohort and group*

hSBA GMR $(95 \% \mathrm{Cl}) \dagger$ for follow-on cohort

\begin{tabular}{|c|c|c|c|c|c|}
\hline Indicator strain & Group 1 & Group 2 & Group 3 & Group 4 (dose 1) & Group 4 (dose 2) \\
\hline H44/76 (fHbp response) & $\begin{array}{c}99(79-125) \\
n=95\end{array}$ & $\begin{array}{c}67(34-135) \\
n=10\end{array}$ & $\begin{array}{c}133(68-258) \\
n=11\end{array}$ & $\begin{array}{c}10(8.2-13) \\
n=175\end{array}$ & $\begin{array}{c}105(94-116) \\
n=175\end{array}$ \\
\hline 5/99 (NadA response) & $\begin{array}{c}70(57-86) \\
n=92\end{array}$ & $\begin{array}{c}51(27-95) \\
n=10\end{array}$ & $\begin{array}{c}55(30-99) \\
n=11\end{array}$ & $\begin{array}{c}29(23-37) \\
n=168\end{array}$ & $\begin{array}{c}299(256-350) \\
n=172\end{array}$ \\
\hline NZ98/254 (PorA response) & $\begin{array}{c}27(21-36) \\
n=93\end{array}$ & $\begin{array}{c}5.96(2.7-13) \\
n=10\end{array}$ & $\begin{array}{c}38(18-81) \\
n=11\end{array}$ & $\begin{array}{c}2.25(1.84-2.75) \\
n=173\end{array}$ & $\begin{array}{c}17(14-19) \\
n=174\end{array}$ \\
\hline M10713 (NHBA response) & $\begin{array}{c}5.24(3.91-7.02) \\
n=88\end{array}$ & $\begin{array}{c}7.06(2.92-17) \\
n=9\end{array}$ & $\begin{array}{c}7.35(3.03-18) \\
n=9\end{array}$ & $\begin{array}{c}2(1.62-2.46) \\
n=158\end{array}$ & $\begin{array}{c}5.12(3.95-6.65) \\
n=161\end{array}$ \\
\hline
\end{tabular}

Note: $\mathrm{Cl}=$ confidence interval, $\mathrm{fHbp}=$ factor $\mathrm{H}$-binding protein, $\mathrm{GMR}=$ geometric mean ratio, $\mathrm{hSBA}=$ human serum bactericidal antibody, $\mathrm{NadA}=\mathrm{Neisserial}$ adhesin $\mathrm{A}, \mathrm{Por} \mathrm{A}=$ porin $\mathrm{A}$ NHBA = Neisseria heparin binding antigen. All participants in the follow-up cohort received 2 previous doses of $4 \mathrm{CMenB}$ vaccine at 12 and 14 mo (Group 1 ), 18 and 20 mo (Group 2 ) or 24 and 26 mo (Group 3) in a previous study. ${ }^{21}$ Group 4 included participants with no previous 4CMenB vaccination.

${ }^{*}$ Values of $n$ are the no. of participants in the group with available results for that response.

TGMR is the ratio of geometric mean titre (GMT) postvaccination to prevaccination. Postvaccination GMT was measured 1 mo after each dose of vaccine, and prevaccination GMT was measured before any doses were given in this study. 95\% Cls for GMRs were computed by taking the anti-log of mean values and the lower and upper limits of the $95 \%$ Cls. 
The accumulating data suggest that waning of bactericidal antibodies against fHbp most commonly occurs more than 12 months after vaccination, whereas waning of anti-PorA anti- bodies occurs almost entirely within 12 months in this age group. Little waning of anti-NadA antibodies was seen up to 26 months after vaccination. This was unlikely to be caused by ongoing

Table 3: Participants with solicited local and systemic adverse events up to day 7 after vaccination for each dose (day of vaccination $=$ day $\mathbf{1}$ )

\begin{tabular}{|c|c|c|c|c|c|}
\hline \multirow[b]{2}{*}{ Local symptom } & \multicolumn{3}{|c|}{ No. (\%) of participants in the follow-up cohort } & \multicolumn{2}{|c|}{$\begin{array}{c}\text { No./n (\%) of participants in the vaccine-naive } \\
\text { cohort: Group } 4\end{array}$} \\
\hline & $\begin{array}{c}\text { Group } 1 \\
n=99\end{array}$ & $\begin{array}{c}\text { Group } 2 \\
n=10\end{array}$ & $\begin{array}{c}\text { Group } 3 \\
n=12\end{array}$ & Dose 1 & Dose 2 \\
\hline Pain, any & $94(94.9)$ & $9(90.0)$ & $11(91.7)$ & $185 / 205(90.2)$ & $157 / 194(80.9)$ \\
\hline Severe* & $19(19.2)$ & $1(10.0)$ & $1(8.3)$ & $27 / 205(13.2)$ & 21/194 (10.8) \\
\hline \multicolumn{6}{|l|}{ Erythema, mm $†$} \\
\hline$\geq 25$ & $21(21.2)$ & $3(30.0)$ & $0(0)$ & 43/204 (21.1) & 34/194 (17.5) \\
\hline$>50$ & $9(9.1)$ & $1(10.0)$ & $0(0)$ & $9 / 204(4.4)$ & 19/194 (9.8) \\
\hline$>100$ & $2(2.0)$ & $0(0)$ & $0(0)$ & $1 / 204(0.5)$ & 0/194 (0) \\
\hline \multicolumn{6}{|l|}{ Induration, $\mathrm{mm} \dagger$} \\
\hline$\geq 25$ & $9(9.1)$ & $1(10.0)$ & $0(0)$ & $26 / 204(12.7)$ & 20/194 (10.3) \\
\hline$>50$ & $1(1.0)$ & $0(0)$ & $0(0)$ & $3 / 204(1.5)$ & $4 / 194(2.1)$ \\
\hline$>100$ & $1(1.0)$ & $0(0)$ & $0(0)$ & $0 / 204(0)$ & $0 / 194(0)$ \\
\hline \multicolumn{6}{|l|}{ Swelling, mm† } \\
\hline$\geq 25$ & $20(20.2)$ & $2(20.0)$ & $4(33.3)$ & $30 / 204(14.7)$ & 24/194 (12.4) \\
\hline$>50$ & $2(2.0)$ & $0(0)$ & $1(8.3)$ & $4 / 204(2.0)$ & 4/194 (2.1) \\
\hline$>100$ & $0(0)$ & $0(0)$ & $0(0)$ & $1 / 204(0.5)$ & $0 / 194(0)$ \\
\hline \multicolumn{6}{|l|}{ Systemic symptom } \\
\hline Change in eating habits, any & $42(42.4)$ & $0(0)$ & $3(25.0)$ & $49 / 203(24.1)$ & $43 / 194(22.2)$ \\
\hline Severe $\ddagger$ & $2(2.0)$ & $0(0)$ & $1(8.3)$ & $3 / 203(1.5)$ & 2/194 (1.0) \\
\hline Sleepiness, any & $52(52.5)$ & $3(30.0)$ & $3(25.0)$ & $74 / 205(36.1) \dagger \dagger$ & $67 / 193(34.7)$ \\
\hline Severe* & $3(3.0)$ & $0(0)$ & $0(0)$ & $5 / 205(2.4)$ & 2/193 (1.0) \\
\hline Vomiting, any & $6(6.1)$ & $2(20.0)$ & $1(8.3)$ & $8 / 205(3.9)$ & $6 / 194(3.1)$ \\
\hline Severe§ & $0(0)$ & $0(0)$ & $0(0)$ & $0 / 205(0)$ & $0 / 194(0)$ \\
\hline Diarrhea, any & $5(5.0)$ & $0(0)$ & $2(16.7)$ & $11 / 204(5.4)$ & $8 / 193(4.1)$ \\
\hline Severeף & $0(0)$ & $0(0)$ & $0(0)$ & $1 / 204(0.5)$ & 0/193 (0) \\
\hline Irritability, any & $53(53.5)$ & $4(40.0)$ & $5(41.7)$ & $67 / 204(32.8)$ & 58/193 (30.0)†† \\
\hline Severe* & $6(6.1)$ & $0(0)$ & $0(0)$ & $8 / 204$ (3.9) & $5 / 193(2.6)$ \\
\hline Headache, any & $20(20.2)$ & $2(20.0)$ & $4(33.3)$ & $25 / 204(12.2)$ & 24/194 (12.4) \\
\hline Severe* & $1(1.0)$ & $0(0)$ & $0(0)$ & $1 / 204(0.5)$ & $1 / 194(0.5)$ \\
\hline Arthralgia, any & $28(28.3)$ & $1(10.0)$ & $6(50.0)$ & $45 / 203(22.2)$ & 40/192 (20.8) \\
\hline Severe* & $10(10.1)$ & $0(0)$ & $1(8.3)$ & $6 / 203(3.0)$ & 2/192 (1.0) \\
\hline Rash & $13(13.1)$ & $0(0)$ & $0(0)$ & $15 / 201(7.5)$ & $10 / 192(5.2)$ \\
\hline \multicolumn{6}{|l|}{ Fever, ${ }^{\star *} \mathrm{C}$} \\
\hline$\geq 38.0$ & $16(16.2)$ & $4(40.0)$ & $5(41.7)$ & 20/204 (9.8) & $16 / 189(8.5)$ \\
\hline$\geq 39.0$ & $0(0)$ & $0(0)$ & $2(16.7)$ & $3 / 204(1.5)$ & $3 / 189(1.6)$ \\
\hline$\geq 40.0$ & $0(0)$ & $0(0)$ & $0(0)$ & $2 / 204(1.0)$ & $0 / 189(0)$ \\
\hline \multicolumn{6}{|l|}{ Fever management } \\
\hline Treatment given & $18(18.2)$ & $4(40.0)$ & $5(41.7)$ & $22 / 204(10.8)$ & 24/193 (12.4) \\
\hline Preventive therapy given & $5(5.0)$ & $2(20.0)$ & $2(16.7)$ & $17 / 204(8.3)$ & 23/192 (12.0) \\
\hline Medical attention sought & $2(2.0)$ & $1(10.0)$ & $0(0)$ & $2 / 204(1.0)$ & $4 / 192(2.1)$ \\
\hline \multicolumn{6}{|c|}{$\begin{array}{l}\text { *Unable to perform daily activity. } \\
\text { †Severe erythema, swelling or induration defined as } \geq 50 \mathrm{~mm} \text {. } \\
\text { †No meals all day. } \\
\text { \$Required intravenous hydration. } \\
\text { "Six or more watery stools per day or required intravenous hydration. } \\
{ }^{*} \text { Axillary temperature. }\end{array}$} \\
\hline
\end{tabular}


boosting by natural exposure, because only $4 \%$ of 4 -year-old children who were vaccine naive had hSBA titres of $1: 5$ or more against strain 5/99. Persistence of the vaccine-induced antiNHBA response was poor because similar proportions of the vaccine-naive and follow-up cohorts had bactericidal antibodies against strain M10713 in our study. The reason for high levels of pre-existing bactericidal antibodies against M10713 in the vaccine-naive cohort is unknown, but it does not appear to impair the vaccine response substantially, with similar postvaccine hSBA GMTs compared with NZ98/254.

In another published study of antibody persistence in this age group in the UK, hSBA titres of 1:4 or more occurred in $0 \%-38 \%$ (depending on target strain) of 3-year-old children after 1 dose of 4 CMenB at 12 months of age, which confirms the need for 2 doses in this age group. ${ }^{26}$ Similar patterns of antigen-dependent differential waning of bactericidal antibodies have been reported after 2 doses of $4 C M e n B$ at 40 months of age. ${ }^{27,28}$ The presence of bactericidal antibodies against one of the vaccine antigens may be sufficient to ensure protection if the protein is expressed at sufficient levels on the bacterial surface. The Meningococcal Antigen Typing System was designed to make this assessment and aims to predict vaccine coverage based on circulating strains. ${ }^{29}$

Similar persistence of antibodies at 4 years of age has been described in children after 3 infant doses of vaccine and a booster in the second year of life. ${ }^{20}$ This suggests that 3 doses in infants and a booster is broadly equivalent to 2 doses in the second year of life with respect to antibody persistence to 4 years. However, this latter regime provides no protection to young infants who have the highest incidence of disease. ${ }^{30}$ Although further doses after 4 years may be required in individuals who are at elevated risk of disease, incidence rates in children who are immunocompetent and aged 5 years or more remain very low until a small increase in adolescence in some populations. ${ }^{30}$

A study conducted in Chile reported that 2 doses of vaccine given to adolescents aged 11-17 years resulted in persistence of SBA of $1: 4$ or more in $75 \%$ or more of participants against all vaccine antigens $18-24$ months after vaccination with 3 doses. ${ }^{31} \mathrm{~A}$ similar study in Australia, Poland and Spain that involved adolescents aged $11-18$ years who received a bivalent fHbp MenB vaccine (rLP2086) reported hSBA titres of 1:4 or more up to 4 years after participants received 3 doses in more than $50 \%$ of participants for 3 of 4 target strains. ${ }^{32}$

Our data suggest that antibody persistence after 2 doses at 24 months is similar to that following vaccination at 12 or 18 months, although the numbers in our study were small. Previous studies have reported substantial waning of bactericidal antibodies by 5 years of age after vaccination at 3 years, ${ }^{27,28}$ which suggests that children receiving 2 doses of $4 C M e n B$ vaccine at 2-3 years of age might require further boosters if ongoing protection is required (e.g., children with splenic dysfunction or complement disorders). Persistence data are needed for children aged 4-10 years through larger studies.

We found that pain at the injection site was the most common local adverse event, with rates similar to those found in previous studies of 4 CMenB vaccine in 3- and 5-year-old children. ${ }^{26-28}$ The rate of fever after vaccination was also similar to previous data. ${ }^{26}$

\section{Limitations}

One limitation of our study is the low proportion of participants in the follow-up cohort from those who completed the previous study (42\% in Group 1, 22\% in Group 2, and 24\% in Group 3). Therefore, there is potential for selection bias because participants who were more tolerant of the previous vaccinations may have been more likely to take part in subsequent studies. The group sizes for participants who received priming doses at 18 and 24 months of age were small, making comparisons among follow-up groups difficult - within this limitation, there were no significant differences between the groups. Further studies would be required to explore differences between schedules in the second year of life.

\section{Conclusion}

Two doses of 4 CMenB vaccine given at $12-24$ months prime the immune system against the vaccine antigens to provide a booster effect after a single dose given 2 years later. The rates at which serum antibody titres to the different vaccine antigens wane vary widely, although the implications of this for vaccine effectiveness have not been established yet. Children receiving their first doses at 2-3 years of age may require further booster doses if ongoing direct protection beyond $4-5$ years is required, such as for those in high-risk groups, although further data in larger cohorts are required to confirm this.

\section{References}

1. Cartwright KA, Stuart JM, Jones DM, et al. The Stonehouse survey: nasopharyngeal carriage of meningococci and Neisseria lactamica. Epidemiol Infect 1987;99:591-601.

2. Brandtzaeg P, van Deuren M. Classification and pathogenesis of meningococcal infections. Methods Mol Biol 2012;799:21-35.

3. Ovstebo R, Hellerud BC, Coureuil M, et al. Pathogenesis of invasive disease. In: Feavers I, Pollard AJ, Sadarangani M, editors. Handbook of meningococcal disease management. Switzerland: Springer; 2016:25-43.

4. Pace D, Pollard AJ. Meningococcal disease: clinical presentation and sequelae. Vaccine 2012;30(Suppl 2):B3-9.

5. Buysse CM, Oranje AP, Zuidema E, et al. Long-term skin scarring and orthopaedic sequelae in survivors of meningococcal septic shock. Arch Dis Child 2009; 94:381-6.

6. Buysse CM, Raat H, Hazelzet JA, et al. Surviving meningococcal septic shock: health consequences and quality of life in children and their parents up to 2 years after pediatric intensive care unit discharge. Crit Care Med 2008;36: 596-602.

7. Buysse CM, Vermunt LC, Raat $\mathrm{H}$, et al. Surviving meningococcal septic shock in childhood: long-term overall outcome and the effect on health-related quality of life. Crit Care 2010;14:R124.

8. Stein-Zamir C, Shoob H, Sokolov I, et al. The clinical features and long-term sequelae of invasive meningococcal disease in children. Pediatr Infect Dis J 2014; 33:777-9.

9. Borg J, Christie D, Coen PG, et al. Outcomes of meningococcal disease in adolescence: prospective, matched-cohort study. Pediatrics 2009;123:e502-9.

10. Judge D, Nadel S, Vergnaud S, et al. Psychiatric adjustment following meningococcal disease treated on a PICU. Intensive Care Med 2002;28:648-50.

11. Sander J, Bay D, Gedde-Dahl TW, et al. Late sequelae after meningococcal disease. A controlled study in young men. NIPH Ann 1984;7:3-11.

12. Borrow R, Abad R, Trotter C, et al. Effectiveness of meningococcal serogroup $C$ vaccine programmes. Vaccine 2013;31:4477-86.

13. MenAfriCar consortium. The diversity of meningococcal carriage across the African meningitis belt and the impact of vaccination with a group A meningococcal conjugate vaccine. J Infect Dis 2015;212:1298-307.

14. Halperin SA, Bettinger JA, Greenwood B, et al. The changing and dynamic epidemiology of meningococcal disease. Vaccine 2012;30(Suppl 2):B26-36.

15. Bettinger JA, Scheifele DW, Le Saux N, et al. The disease burden of invasive meningococcal serogroup B disease in Canada. Pediatr Infect Dis J 2013;32:e20-5. 
16. Drysdale SB, Pollard AJ. Group B meningococcal vaccine science and policy. J Infect 2015;71(Suppl 1):S15-20.

17. Watson PS, Turner DP. Clinical experience with the meningococcal B vaccine, Bexsero $\left({ }^{\circledR}\right)$ : prospects for reducing the burden of meningococcal serogroup $B$ disease. Vaccine 2016;34:875-80.

18. Bexsero. Summary of product characteristics. London (UK): European Medicines Agency; 2012. Available: www.ema.europa.eu/docs/en_GB/document_library/ EPAR_-_Product_Information/human/002333/WC500137881.pdf (accessed 2016 Feb. 14).

19. Basta NE, Mahmoud AA, Wolfson J, et al. Immunogenicity of a meningococcal B vaccine during a university outbreak. N Engl J Med 2016;375:220-8.

20. Iro MA, Snape MD, Voysey M, et al. Persistence of bactericidal antibodies following booster vaccination with $4 \mathrm{CMenB}$ at 12,18 or 24 months and immunogenicity of a fifth dose administered at 4 years of age - a phase 3 extension to a randomised controlled trial. Vaccine 2017;35:395-402.

21. Snape MD, Voysey M, Biostat M, et al. Persistence of bactericidal antibodies following infant serogroup $B$ meningococcal immunization and booster dose response at 12, 18 or 24 months of age. Pediatr Infect Dis J 2016;35:e113-23.

22. Snape MD, Dawson $T$, Oster $P$, et al. Immunogenicity of two investigational serogroup $B$ meningococcal vaccines in the first year of life: a randomized comparative trial. Pediatr Infect Dis J 2010;29:e71-9.

23. Frasch CE, Borrow R, Donnelly J. Bactericidal antibody is the immunologic surrogate of protection against meningococcal disease. Vaccine 2009;27(Suppl 2):B112-6.

24. Parikh SR, Andrews NJ, Beebeejaun K, et al. Effectiveness and impact of a reduced infant schedule of 4 CMenB vaccine against group $B$ meningococcal disease in England: a national observational cohort study. Lancet 2016;388: 2775-82.
25. Vesikari T, Prymula R, Merrall E, et al. Meningococcal serogroup B vaccine (4CMenB): Booster dose in previously vaccinated infants and primary vaccination in toddlers and two-year-old children. Vaccine 2015;33:3850-8.

26. Snape MD, Saroey P, John TM, et al. Persistence of bactericidal antibodies following early infant vaccination with a serogroup $B$ meningococcal vaccine and immunogenicity of a preschool booster dose. CMAJ 2013;185:E715-24.

27. McQuaid F, Snape MD, John TM, et al. Persistence of bactericidal antibodies to 5 years of age after immunization with serogroup $B$ meningococcal vaccines at 6, 8, 12 and 40 months of age. Pediatr Infect Dis J 2014;33:760-6.

28. McQuaid F, Snape MD, John TM, et al. Persistence of specific bactericidal antibodies at 5 years of age after vaccination against serogroup $B$ meningococcus in infancy and at 40 months. CMAJ 2015;187:E215-23.

29. Vogel U, Taha MK, Vazquez JA, et al. Predicted strain coverage of a meningococcal multicomponent vaccine $(4 \mathrm{CMenB})$ in Europe: a qualitative and quantitative assessment. Lancet Infect Dis 2013;13:416-25.

30. Ciaravino G, Högberg LD, Ködmön C, et al. EDCD Surveillance report: surveillance of invasive bacterial diseases in Europe, 2012. Invasive pneumococcal disease, invasive Haemophilus influenzae disease and invasive meningococcal disease. Stockholm: European Centre for Disease Prevention and Control; 2015. Available: http://ecdc.europa.eu/en/publications/Publications/Surveillance \%20of\%20IBD\%20in\%20Europe\%202012.pdf (accessed 2016 Oct. 8).

31. Santolaya ME, O'Ryan M, Valenzuela MT, et al. Persistence of antibodies in adolescents 18-24 months after immunization with one, two, or three doses of 4CMenB meningococcal serogroup B vaccine. Hum Vaccin Immunother 2013;9:2304-10.

32. Marshall HS, Richmond PC, Beeslaar J, et al. Meningococcal serogroup B-specific responses after vaccination with bivalent rLP2086: 4 year follow-up of a randomised, single-blind, placebo-controlled, phase 2 trial. Lancet Infect Dis 2017;17:58-67.
Competing interests: Manish Sadarangani has received a grant from Novartis during the conduct of this study (Novartis Vaccines Division was subsequently acquired by the GSK group of companies) and a grant for investigator-initiated research studies outside the submitted work from Pfizer. Manish Sadarangani has also received payment as site investigator/co-investigator for multicentre trials from Variation Biotechnologies and Merck. Matthew Snape has received a grant from Novartis during the conduct of this study (Novartis Vaccines Division was subsequently acquired by GSK group of companies) and grants paid to his institution for work as an investigator on clinical trials from Novartis Vaccines and Diagnostics (subsequently acquired by GSK group of companies), GSK group of companies, Pfizer, Janssen, Medlmmune, Alios BioPharma and Ablynx. He has also received support for travel and accommodation to attend international conferences from GSK group of companies, and he has received support paid to his institution as a member of the advisory board for Sanofi-Pasteur MSD and as a consultant for Medlmmune. Matthew Snape receives salary support from the National Institute for Health Research Oxford Biomedical Research Centre and is a Jenner Investigator. Adam Finn has received grants paid to his institution from Novartis, GSK group of companies, Pfizer and Sanofi-Pasteur MSD for studies outside the submitted work, and a grant from Novartis (Novartis Vaccines Division was subsequently acquired by the GSK group of companies) for the submitted work. Owing to his membership on the Joint Committee on Vaccination and Immunisation for the United Kingdom Department of Health,
Adam Finn no longer gives lectures or undertakes advisory work for industry, either paid or unpaid. Paul Heath has received grants paid to his institution from GSK group of companies, Sanofi-Pasteur MSD and Novartis for studies outside the submitted work, and a grant from Novartis for the submitted work. Gianni Bona has received lecture fees and nonfinancial support for clinical trials during the conduct of this study from Novartis Vaccines and Diagnostics, and support from Novartis Vaccines and Diagnostics, GSK group of companies, Sanofi-Pasteur and Pfizer as an investigator for clinical trials outside the submitted work. Susanna Esposito has received a grant paid to her institution from Novartis Vaccines and Diagnostics; grants from Novartis Vaccines and Diagnostics, GSK group of companies, Pfizer, Sanofi-Aventis and Sanofi-Pasteur MSD; and personal fees (in the form of consultant and speaker fees, and reimbursements for travel and accommodation) from Novartis Vaccines and Diagnostics, GSK group of companies, Pfizer and MedImmune. Javier Diez-Domingo has received reimbursement for being a member of the advisory committees for GSK group of companies and Pfizer, as well as travel support from Pfizer. Roman Prymula received a grant for the submitted work from Novartis, and has received grants from Novartis, Sanofi-Pasteur MSD and GSK group of companies outside the submitted work. Mildred Iro has received nonfinancial support for travel to conferences from GSK group of companies. Andrew Pollard has received a grant from Novartis during the conduct of this study, and grants from Pfizer and Okairos in the past 36 months. His department received unrestricted educational grants from Pfizer,
GSK group of companies and AstraZeneca in July 2016, and Gilead, MSD, GSK group of companies and AstraZeneca in June 2017 for a course on Infection and immunity in children. Andrew Pollard is the chair of UK Department of Health's Joint Committee on Vaccination and Immunisation, and the Vaccines Scientific Advisory group of the European Medicines Agency, and is a member of the World Health Organization's Strategic Advisory Group of Experts (SAGE). Adefowope Odueyungbo was a GSK employee at the time the study was conducted (formerly Novartis Vaccines and Diagnostics). Daniela Toneatto declares that she is employed by and holds shares in the GSK group of companies. Andrew Pollard, Paul Heath, Matthew Snape, Manish Sadarangani and Adam Finn do not receive any personal remuneration from vaccine manufacturers.

This article has been peer reviewed.

Affiliations: Oxford Vaccine Group, Department of Paediatrics (Sadarangani, Sell, Iro, Snape, Voysey, Pollard), University of Oxford, and the NIHR Oxford Biomedical Research Centre, Oxford, UK; Vaccine Evaluation Center (Sadarangani), BC Children's Hospital Research Institute, The University of British Columbia, Vancouver, BC; Nuffield Department of Primary Care Health Sciences (Voysey), University of Oxford, Oxford, UK; Bristol Children's Vaccine Centre (Finn), School of Clinical Sciences, University of Bristol, Bristol, UK; St. George's Vaccine Institute (Heath), University of London, London, UK; Azienda Ospedaliero-Universitaria Maggiore della Carità (Bona), Clinica Pediatrica, Novara, Italy; Pediatric Highly Intensive Care Unit (Esposito), 
Università degli Studi di Milano, Fondazione IRCCS Ca' Granda Ospedale Maggiore Policlinico, Milan, Italy; Vaccine Research Area (DiezDomingo), Fundación para el Fomento de la Investigación Sanitaria y Biomédica (FISABIO), Valencia, Spain; Charles University Prague, School of Medicine, Department of Social Sciences (Prymula), Hradec Kralove, Czech Republic; Novartis Vaccines and Diagnostics Inc. (Odueyungbo), Cambridge, Mass.; Hoffmann-La Roche Limited (Odueyungbo), Mississauga, Ont.; GSK (Toneatto), Siena, Italy

Contributors: Matthew Snape, Daniela Toneatto and Andrew Pollard conceived and designed the study; Tim Sell, Mildred Iro, Matthew Snape, Adam Finn, Paul Heath, Gianni Bona, Susanna Esposito, Javier Diez-Domingo, Roman Prymula conducted the study and acquired the data; Daniela Toneatto, Mildred Iro, Matthew Snape, Andrew Pollard, Tim Sell, Manish Sadarangani, Merryn Voysey, Adefowope Odueyungbo analyzed and interpreted the data; and Manish Sadarangani and Tim Sell drafted the work. All of the authors revised the manuscript critically for important intellectual content, gave final approval of the version to be published and agreed to be accountable for all aspects of the work.

Funding: This study was sponsored by Novartis Vaccines Division. On Mar. 2, 2015, the Novartis Vaccines non-influenza segment was acquired by the GlaxoSmithKline group of companies. With the lead investigators, the sponsor was involved in the design of the study, as well as analysis of the data, and review and comment on the manuscript. Data collection was undertaken by the study investigators' teams. Editorial control of the manu- script was assigned to the University of Oxford. The sponsor conducted the primary analysis of the data before an independent validation with full access to all data at the University of Oxford by Merryn Voysey.

Data sharing: All rights and titles on the product, data and results generated during this clinical study were acquired by GSK from Novartis. The results summary for this study (Legacy Novartis study no. V72P12E2 NCT01717638) is available on the Novartis Clinical Trials Results website and can be accessed at https://www.novctrd.com/. For interventional studies that evaluate GSK medicines, anonymized patient-level data will be made available to independent researchers, subject to review by an independent panel, at http://www.clinicalstudydatarequest.com/ within 6 months of publication. To protect the privacy of patients and individuals involved in our studies, GSK does not publically disclose patient- level data.

Disclaimer: The views presented in this manuscript do not necessarily represent the views of the Department of Health or the Joint Committee on Vaccination and Immunisation.

Acknowledgements: The authors thank all of the participants, their families and the study staff at the research centres in the participating countries for contributing to this study. The authors also thank ludit-Hajnal Filip (XPE Pharma \& Science, Wavre, Belgium c/o GSK) for publication coordination.

*The European Men B Vaccine Study Group: Sarah Kelly RN, Danielle Campbell RN, Oxford Vaccine Group, Department of Paediatrics, University of Oxford, Oxford, UK; Sandra
Dymond RN, Bristol Children's Vaccine Centre, School of Clinical Sciences, University of Bristol, Bristol, UK; Alison Kent MD, St. George's Vaccine Institute, University of London, London, UK; Maurizio De Martino MD, Leila Bianchi MD, Carlotta Montagnani MD, Anna Meyer Children's University Hospital, Department of Health Sciences, University of Florence, Florence, Italy; Carlo Giaquinto MD, Department of Pediatrics, University of Padua, Padua, Italy; Benedetta Ghezzi PhD, Igor Kohl $\mathrm{PhD}$, Simone Inzillo, Linda Kasim MSc, Lucie Hlavata MD, Laura Lulli MSc, Harry Wisse, Sheena Gomez PhD, Vasundhara Dindore MD, GSK (formerly Novartis Vaccines and Diagnostics Srl), Siena, Italy; Erika Pozzi MD, Silvia Parlamento MD, Azienda OspedalieroUniversitaria Maggiore della Carità - Clinica Pediatrica, Novara, Italy; Valentina Montinaro MSc, Fondazione IRCCS Cà Granda Ospedale Maggiore Policlinico, Università degli Studi di Milano, Milan, Italy; Miguel TortajadaGirbés MD, Centro de Salud de Catarroja, Catarroja, Valencia, Spain; Federico MartinónTorres MD, Lorenzo Redondo Collazo MD, Hospital Clinico Universitario de Santiago de Compostela, Santiago de Compostela, Spain; Roman Chlíbek MD PhD, University of Defense, Faculty of Military Health Sciences, Vaccination Center, Hradec Králové, Czech Republic; Daniel Dražan MD, Samostatna ordinace praktickeho lekare pro deti a dorost, Jindřichův Hradec, Czech Republic.

Accepted: June 12, 2017

Correspondence to: Matthew Snape, matthew.snape@paediatrics.ox.ac.uk 